\title{
Global Self-similar Solutions of a Class of Nonlinear Schrödinger Equations
}

\author{
Yaojun YE \\ Department of Mathematics and Information Science, Zhejiang University of Science and Technology, \\ Hangzhou 310023, China
}

Correspondence should be addressed to Yaojun YE, yeyaojun2002@yahoo.com.cn

Received 11 November 2007; Accepted 4 March 2008

Recommended by Thomas Bartsch

For a certain range of the value $p$ in the nonlinear term $|u|^{p} u$, in this paper, we mainly study the global existence and uniqueness of global self-similar solutions to the Cauchy problem for some nonlinear Schrödinger equations using the method of harmonic analysis.

Copyright ( 2008 Yaojun YE. This is an open access article distributed under the Creative Commons Attribution License, which permits unrestricted use, distribution, and reproduction in any medium, provided the original work is properly cited.

\section{Introduction}

This paper is devoted to study the initial value problem for the nonlinear Schrödinger equation

$$
\begin{aligned}
i u_{t}+(-\Delta)^{m} u & =\lambda|u|^{p} u, \quad x \in R^{n}, t \in R^{+}, \\
u(x, 0) & =f(x), \quad x \in R^{n},
\end{aligned}
$$

where $\lambda \in R, p>0$ are constants, $m \geq 1$ is a positive integer, $u(t, x)$ is a complex-valued function defined in $R^{+} \times R^{n}$, the initial value $f(x)$ is a complex-valued function defined in $R^{n}$.

When $m=1,(1.1)$ is a classical nonlinear Schrödinger equation of the second order:

$$
i u_{t}-\Delta u=\lambda|u|^{p} u .
$$

For the Cauchy problem of (1.2), the existence and the scattering theorem of solutions have been studied extensively by many authors with various methods and techniques [1-5], Cazenave and Weissler [6] (also Ribaud and Youssfi [7]) established existence of global selfsimilar solutions by introducing new function space. When $m \geq 1$, Pecher and von Wahl [8] established the existence of classical solution of the Cauchy problem (1.1) employing the related $L^{p}$ estimate of the elliptic equation and the compact method. Sjölin and Sjögren in $[9,10]$ 
recently discussed the local smooth effect of solutions of the Cauchy problem (1.1) applying the Strichartz estimate in the nonhomogeneous Sobolev space. In [11], by constructing a timeweighted space and using the contractive mapping method, the author established global solutions of the problem (1.1) in the possible range of $p$, and further got the continuous dependence of the solution on the initial value together with its strong decay estimate. In addition, there are also much more efforts working for studying the scattering theorem and the existence of global strong solutions of the problem (1.1) $[12,13]$. In this paper, we mainly investigate the existence of global self-similar solutions basing on the existence and uniqueness of global solution for the Cauchy problem (1.1).

In the following discussion, we suppose that $p$ satisfies

$$
p_{0}<p<\frac{4 m}{n-2 m}, \quad n>2 m, p_{0}<p<+\infty, n \leq 2 m,
$$

where $p_{0}$ is a positive solution of the equation $n x^{2}+(n-2 m) x-4 m=0$, which also can be interpreted as a positive integer satisfying $(p+2) /(p+1)=n p / 2 m$. In fact, condition (1.3) is equivalent to

$$
\frac{p+2}{p+1}<\frac{n p}{2 m}<p+2
$$

For $p$ which satisfies (1.3) or (1.4), let

$$
\theta=\frac{4 m-(n-2 m) p}{2 m p(p+2)}
$$

then we may introduce our work space $X$ as follows. Let $X$ be a space consisting of all Bochner measurable functions:

$$
u(t):(0,+\infty) \longrightarrow L^{p+2}\left(R^{n}\right),
$$

such that

$$
\|u\|_{X}=\sup _{t>0} t^{\theta}\|u(t)\|_{p+2}<+\infty
$$

In order to prove our main result, we should transform the Cauchy problem (1.1) into the following equivalent integral equation:

$$
u(t)=S(t) f(x)-i \lambda \int_{0}^{t} S(t-s)\left(|u(s)|^{p} u(s)\right) d s,
$$

where $S(t)=e^{i(-\Delta)^{m} t}=\mathcal{F}^{-1}\left(e^{i|\xi|^{2 m} t} \mathcal{F} \cdot\right)$ is a free group produced by the free Schrödinger equation $i v_{t}+(-\Delta)^{m} v=0$. Besides, we denote, respectively, by $\mathcal{F}$ and $\mathcal{F}^{-1}$ the Fourier transformation and the inverse Fourier transformation with respect to the space variables.

For convenience, we provide some useful symbols. $L^{r}\left(R^{n}\right)$ denotes the usual Lebesgue space on $R^{n}$ with the norm $\|\cdot\|_{r}, 1 \leq r \leq+\infty$. For any $q>0, q^{\prime}$ stands for the dual to $q$, that is, 
$(1 / q)+\left(1 / q^{\prime}\right)=1$. $C$ which may be different when appeared every time is a constant depending on the dimension or any other constant.

In the end, we will review the definition of the homogeneous Besov space, the details on the properties, and the embedding theorems reference $[1,14]$.

Let $\widehat{\varphi}(\xi) \in \mathcal{S}$ be a symmetric Bump function with real values satisfying the conditions $\widehat{\varphi}(\xi)=1,|\xi| \leq 1, \widehat{\varphi}(\xi)=0,|\xi|>2$, then

$$
\widehat{\varphi}_{j}(\xi)=\widehat{\varphi}\left(2^{-j} \xi\right), \quad \widehat{\varphi}_{j}(\xi)=\widehat{\psi}\left(2^{-j} \xi\right)=\widehat{\varphi}\left(2^{-j} \xi\right)-\widehat{\varphi}\left(2^{-j+1} \xi\right), \quad j \in Z
$$

are also symmetric Bump functions. Denote by $\Delta_{j}$ and $S_{j}$ the convolution operator of $\widehat{\psi}_{j}(\xi)$ and $\widehat{\varphi}_{j}(\xi)$, respectively, that is,

$$
\Delta_{j} f=\mathcal{F}^{-1} \widehat{\psi}_{j} \mathscr{F} f=\psi_{j} * f, \quad S_{j} f=\mathcal{F}^{-1} \widehat{\varphi}_{j} \mathscr{F} f=\varphi_{j} * f \quad \forall j \in Z .
$$

If $s \in R, 1 \leq p \leq+\infty, 1 \leq q<+\infty$, then

$$
\dot{B}_{p}^{s, q}=\left\{f \in S^{\prime}:\|f\|_{\dot{B}_{p}^{s, q}}=\left[\sum_{j \in Z} 2^{s j q}\left\|\Delta_{j} f\right\|_{p}^{q}\right]^{1 / q}<+\infty\right\}
$$

is called a homogeneous Besov space and

$$
\dot{B}_{p}^{s, \infty}=\left\{f \in \mathcal{S}^{\prime}:\|f\|_{\dot{B}_{p}^{s, \infty}}=\sup _{j \in Z} 2^{j s}\left\|\Delta_{j} f\right\|_{p}<+\infty\right\} .
$$

\section{Lemmas and main results}

The linear Schrödinger group $S(t)=e^{i(-\Delta)^{m} t}$ satisfies the following $L^{q^{\prime}}-L^{q}$ estimate $[14,15]$ :

$$
\|S(t) f(x)\|_{q}=\left\|\mathcal{F}^{-1}\left(e^{i|\xi|^{2 m} t} \mathcal{F} f\right)\right\|_{q} \leq C|t|^{-(n / m)(1 / 2-1 / q)}\|\varphi\|_{q^{\prime}}, \quad 2 \leq q \leq+\infty \forall t>0 .
$$

We first provide two lemmas that may be useful in in the following.

Lemma 2.1. Let $f(x)=\Omega(x /|x|)|x|^{-2 m / p}, \theta=(4 m-(n-2 m) p) / 2 m p(p+2)$, then $\left\|u_{0}\right\|_{X}=$ $\|S(t) f\|_{X}=\|S(1) f\|_{p+2}$.

Proof. According to the property of the Fourier transformation and $f(x)=\lambda^{2 m / p} f(\lambda x)$, we get

$$
S(t) f=\lambda^{2 m / p}\left[S\left(\lambda^{2 m} t\right)\right] f(\lambda x) \quad \forall \lambda>0 .
$$

Let $\lambda=1 / \sqrt[2 m]{t}$, then

$$
S(t) f=t^{-1 / p}[S(1)] f\left(\frac{x}{\sqrt[2 m]{t}}\right)
$$

Thus

$$
t^{\theta}\|S(t) f\|_{p+2}=t^{\theta-1 / p}\left\|[S(1) f]\left(\frac{x}{\sqrt[2 m]{t}}\right)\right\|_{p+2}=t^{\theta-1 / p+n / 2 m(p+2)}\|S(1) f\|_{p+2} .
$$


Since

$$
\theta-\frac{1}{p}+\frac{n}{2 m(p+2)}=0
$$

It is easy to see that from (2.4) and (2.5),

$$
\sup _{t>0} t^{\theta}\|S(t) f\|_{p+2}=\|S(1) f\|_{p+2}
$$

namely,

$$
\left\|u_{0}\right\|_{X}=\|S(t) f\|_{X}=\|S(1) f\|_{p+2} .
$$

Lemma 2.2. Let $\Omega \in C^{k}\left(S^{n-1}\right), k \geq 0, f(x)=\Omega(x /|x|)|x|^{-d}, 0<d<n$, then

$$
\left|\Delta_{0}(f)(x)\right| \leq C\|\Omega\|_{C^{k}}(1+|x|)^{-k-d} .
$$

The detailed proof can be referred to [16].

In order to prove the main results, we need the following known theorems [11].

Theorem 2.3 (existence of global solutions). Suppose that $p$ satisfies (1.3) or (1.4), $\theta=(4 m-(n-$ $2 m) p) /(2 m p(p+2)), u_{0}(t, x)=[S(t) f](x)$ if there is $\varepsilon>0$, such that

$$
\left\|u_{0}\right\|_{X}=\|S(t) f\|_{X} \leq \varepsilon
$$

then the Cauchy problem (1.1) has a unique solution $u(x, t) \in X$ which satisfies $\|u\|_{X} \leq 2 \varepsilon$.

Theorem 2.4 (the continuous dependence of the solution on the initial value). Suppose that $f(x)$ and $g(x)$ both satisfy the condition (2.9), $u, v$ are two solutions of the Cauchy problem (1.1) corresponding to the initial value $f(x)$ and $g(x)$, then

$$
\|u-v\|_{X} \leq C\|S(t)(f-g)\|_{X}
$$

In addition, if

$$
\sup _{t>0} t^{\theta}(1+t)^{\delta}\|S(t)(f-g)\|_{p+2}<+\infty
$$

then

$$
\|u-v\|_{p+2} \leq C t^{-\theta}(1+t)^{-\delta}
$$

where $(p+1) \theta+\delta<1, \delta>0$.

In this paper, our object is to study the global self-similar solutions of the Cauchy problem (1.1). At first, we introduce the definition of the self-similar solution. 
Definition 2.5. Suppose that $u(t, x)$ is a solution of the Cauchy problem (1.1), if

$$
u(t, x)=u_{\lambda}(t, x)=\lambda^{2 m / p} u\left(\lambda^{2 m} t, \lambda x\right) \quad \forall \lambda>0,
$$

then $u(t, x)$ is called the self-similar solution of the problem (1.1).

One easily knows from the above definition that $u_{\lambda}(t, x)=\lambda^{2 m / p} u\left(\lambda^{2 m} t, \lambda x\right) \forall \lambda>0$ is a solution of the problem (1.1) which satisfies the initial value $\lambda^{2 m / p} f(\lambda x)$, provide that $u(t, x)$ is just a solution of the Cauchy problem (1.1).

Now, we give our main result.

Theorem 2.6. Let $p$ satisfy (1.3) or (1.4), $\theta=(4 m-(n-2 m) p) / 2 m p(p+2), \Omega \in C^{n}\left(S^{n-1}\right)$, and

$$
f(x)=\frac{\Omega(x /|x|)}{|x|^{2 m / p}}
$$

$u_{0}(t, x)=S(t) f(x)$, then

$$
\left\|u_{0}\right\|_{X} \leq C\|\Omega\|_{C^{n}}
$$

In particular, if existing $\varepsilon^{\prime}=\varepsilon / C>0$ such that $\|\Omega\|_{C^{n}} \leq \varepsilon^{\prime}$, then there exists a unique self-similar solution of (1.1) with the initial value (2.14).

\section{The proof of main result}

To prove Theorem 2.6, we should provide the following two propositions.

Proposition 3.1. Let

$$
f(x)=\Omega\left(\frac{x}{|x|}\right)|x|^{-2 m / p}, \quad \theta=\frac{4 m-(n-2 m) p}{2 m p(p+2)},
$$

then

$$
\left\|u_{0}\right\|_{X}=\|S(t) f\|_{X} \leq C\left\|\Delta_{0}(f)\right\|_{(p+2)^{\prime}} .
$$

Proof. By Lemma 2.1, we only illustrate that the following inequality is valid:

$$
\|S(1) f\|_{p+2} \leq C\left\|\Delta_{0}(f)\right\|_{(p+2)^{\prime}}
$$

It follows that from the embedding $\dot{B}_{p+2}^{0,1} \hookrightarrow \dot{H}_{p+2}^{0}=L^{p+2}$, it is necessary to prove

$$
\|S(1) f\|_{\dot{B}_{p+2}^{0,1}} \leq C\left\|\Delta_{0}(f)\right\|_{(p+2)^{\prime}} .
$$

Denote $F=S(1) f$, and then $F$ can be decomposed as follows:

$$
F=F_{1}+F_{2}, \quad F_{1}=S(1)(\varphi * f), \quad F_{2}=S(1)\left(\mathcal{F}^{-1}(1-\widehat{\varphi}) * f\right),
$$

where $\varphi$ is referred in the introduction. 
Making use of the estimate (2.1) and noting that $\Delta_{j} \mathcal{F}^{-1}(1-\widehat{\varphi})=0$ for all $j \leq-1$, then we have

$$
\begin{aligned}
\left\|F_{2}\right\|_{\dot{B}_{p+2}^{0,1}} & =\sum_{j \in Z}\left\|\Delta_{j} F_{2}\right\|_{p+2}=\sum_{j \in Z}\left\|S(1) \Delta_{j}\left(\mathcal{F}^{-1}((1-\widehat{\varphi}) * f)\right)\right\|_{p+2} \\
& \leq \sum_{j \in Z}\left\|\Delta_{j}\left(\mathcal{F}^{-1}((1-\widehat{\varphi}) * f)\right)\right\|_{(p+2)^{\prime}} \\
& =\sum_{j \geq 0}\left\|\Delta_{j}\left(\mathcal{F}^{-1}((1-\widehat{\varphi}) * f)\right)\right\|_{(p+2)^{\prime}} \\
& =\sum_{j \geq 0}\left\|\widetilde{\Delta}_{j} \Delta_{j}\left(\mathcal{F}^{-1}((1-\widehat{\varphi}) * f)\right)\right\|_{(p+2)^{\prime}}
\end{aligned}
$$

where $\widetilde{\Delta}_{j}=\sum_{l=-1}^{l=1} \Delta_{j+l}$.

For $l= \pm 1,0$, we have

$$
\left\|\Delta_{j+l} \mathcal{F}^{-1}(1-\widehat{\varphi})\right\|_{1}=\left\|\mathcal{F}^{-1}\left(\widehat{\varphi}_{j+l}(1-\widehat{\varphi})\right)\right\|_{1} \leq\left\|\psi_{j+l}\right\|_{1}+\left\|\psi_{j+l} * \varphi\right\|_{1} .
$$

Since $\psi_{j+l}(x)=2^{(j+l) n} \psi_{0}\left(2^{j+l} x\right)$, then $\left\|\psi_{j+l}\right\|_{1}=\left\|\psi_{0}\right\|_{1}$. Thus, it follows that from the Young inequality

$$
\left\|\Delta_{j+l} \mathcal{F}^{-1}(1-\widehat{\varphi})\right\|_{1} \leq C\left\|\psi_{j+l}\right\|_{1}\left(1+\|\varphi\|_{1}\right) \leq C .
$$

Besides, as $f(\lambda x)=\lambda^{-2 m / p} f(x)$, so that

$$
\begin{aligned}
\Delta_{j} f(x) & =\psi_{j} * f(x)=\int_{R^{n}} \psi_{j}(x-y) f(y) d y \\
& =2^{j n} \int_{R^{n}} \psi_{0}\left(2^{j} x-2^{j} y\right) f(y) d y \\
& =\int_{R^{n}} \psi_{0}\left(2^{j} x-z\right) f\left(2^{-j} z\right) d z \\
& =2^{j(2 m / p)}\left(\psi_{0} * f\right)\left(2^{j} x\right)=2^{j(2 m / p)} \Delta_{0} f\left(2^{j} x\right) .
\end{aligned}
$$

Therefore,

$$
\left\|\Delta_{j} f\right\|_{(p+2)^{\prime}}=2^{j(2 m / p)}\left\|\Delta_{0} f\left(2^{j} \cdot\right)\right\|_{(p+2)^{\prime}}=2^{j\left(2 m / p-n /(p+2)^{\prime}\right)}\left\|\Delta_{0} f\right\|_{(p+2)^{\prime}} .
$$

By (3.6) together with the Young inequality, we obtain

$$
\left\|F_{2}\right\|_{\dot{B}_{p+2}^{0,1}} \leq \sum_{j \geq 0}\left\|\widetilde{\Delta}_{j}\left(\mathcal{F}^{-1}(1-\widehat{\varphi})\right)\right\|_{1}\left\|\Delta_{j} f\right\|_{(p+2)^{\prime}} \leq C\left\|\Delta_{0} f\right\|_{(p+2)^{\prime}} \sum_{j \geq 0} 2^{j\left(2 m / p-n /(p+2)^{\prime}\right)} .
$$

We know that from the left side of the inequality (1.4),

$$
\frac{2 m}{p}-\frac{n}{(p+2)^{\prime}}<0
$$


It yields from (3.11) that

$$
\left\|F_{2}\right\|_{B_{p+2}^{0,1}} \leq C\left\|\Delta_{0} f\right\|_{(p+2)^{\prime}} .
$$

On the other hand, $\Delta_{j} \varphi=0$ for $j \geq 2$, thus

$$
\left\|F_{1}\right\|_{B_{p+2}^{0,1}}=\sum_{j \in Z}\left\|\Delta_{j} F_{1}\right\|_{p+2}=\sum_{j \leq 1}\left\|S(1) \Delta_{j}(\varphi * f)\right\|_{p+2} .
$$

It follows that by the Young inequality,

$$
\left\|S(1) \Delta_{j}(\varphi * f)\right\|_{p+2} \leq\|S(1) \varphi\|_{1}\left\|\Delta_{j} f\right\|_{p+2} .
$$

We get that from (3.15) and $\left\|\Delta_{j} f\right\|_{p+2}=2^{j(2 m / p-n /(p+2))}\left\|\Delta_{0} f\right\|_{p+2}$,

$$
\left\|S(1) \Delta_{j}(\varphi * f)\right\|_{p+2} \leq C 2^{j(2 m / p-n /(p+2))}\left\|\Delta_{0} f\right\|_{p+2} .
$$

Correspondingly,

$$
\left\|F_{1}\right\|_{\dot{B}_{p+2}^{0,1}} \leq C\left\|\Delta_{0} f\right\|_{p+2} \sum_{j \leq 1} 2^{j(2 m / p-n /(p+2))} .
$$

The right side of (1.4) shows that $2 m / p-n /(p+2)>0$, consequently

$$
\left\|F_{1}\right\|_{\dot{B}_{p+2}^{0,1}} \leq C\left\|\Delta_{0} f\right\|_{p+2}
$$

From $(p+2)^{\prime} \leq p+2$ and the Bernstein inequality, we get

$$
\left\|F_{1}\right\|_{\dot{B}_{p+2}^{0,1}} \leq C\left\|\Delta_{0} f\right\|_{p+2} \leq C\left\|\Delta_{0} f\right\|_{(p+2)^{\prime}}
$$

Combining (3.13) with (3.19), we have

$$
\|F\|_{\dot{B}_{p+2}^{0,1}} \leq C\left\|\Delta_{0} f\right\|_{(p+2)^{\prime}}
$$

The proof of Proposition 3.1 is finished.

Proposition 3.2. Let $\Omega \in C^{n}\left(S^{n-1}\right), f(x)=\Omega(x /|x|)|x|^{-2 m / p}$, then

$$
\left\|\Delta_{0} f\right\|_{(p+2)^{\prime}} \leq C\|\Omega\|_{C^{n}} .
$$

Proof. Since $(p+2)^{\prime} \geq 1$, then $(n+2 m / p)(p+2)^{\prime}>n$. Accordingly, we obtain by Lemma 2.2 that

$$
\begin{aligned}
\left\|\Delta_{0} f\right\|_{(p+2)^{\prime}}^{(p+2)^{\prime}} & =\int_{R^{n}}\left|\Delta_{0} f(x)\right|^{(p+2)^{\prime}} d x \\
& \leq C \int_{R^{n}}\|\Omega\|_{C^{n}}^{(p+2)^{\prime}}(1+|x|)^{-(n+2 m / p)(p+2)^{\prime}} d x \\
& \leq C\|\Omega\|_{C^{n}}^{(p+2)^{\prime}} \int_{0}^{+\infty}(1+r)^{-(n+2 m / p)(p+2)^{\prime}+n-1} d r \\
& \leq C\|\Omega\|_{C^{n}}^{(p+2)^{\prime}}
\end{aligned}
$$


which implies that

$$
\left\|\Delta_{0} f\right\|_{(p+2)^{\prime}} \leq C\|\Omega\|_{C^{n}}
$$

The proof is concluded.

Now, we are ready to prove Theorem 2.6.

Proof. For

$$
f(x)=\Omega\left(\frac{x}{|x|}\right)|x|^{-2 m / p},
$$

we have from Proposition 3.1

$$
\|S(t) f\|_{X} \leq C\left\|\Delta_{0}(f)\right\|_{(p+2)^{\prime}}
$$

However, noting that $\Omega \in C^{n}\left(S^{n-1}\right)$ as well as Proposition 3.2, we get

$$
\left\|\Delta_{0}(f)\right\|_{(p+2)^{\prime}} \leq C\|\Omega\|_{C^{n}}
$$

Then, it follows from (3.25) and (3.26) that

$$
\left\|u_{0}\right\|_{X}=\|S(t) f\|_{X} \leq C\|\Omega\|_{C^{n}}
$$

Choosing $\varepsilon^{\prime}=\varepsilon / C>0$, then we have $\left\|u_{0}\right\|_{X} \leq \varepsilon$ for any $\|\Omega\|_{C^{n}} \leq \varepsilon^{\prime}$. From Theorem 2.3, we conclude that there is a unique global solution $u(x, t)$ of the equation in (1.1) with the initial value (2.14). Besides,

$$
\lambda^{2 m / p} f(\lambda x)=\Omega\left(\frac{x}{|x|}\right)|x|^{-2 m / p}=f(x)
$$

which gives that by uniqueness

$$
u(x, t)=\lambda^{2 m / p} u\left(\lambda x, \lambda^{2 m} t\right)
$$

Thus, $u(x, t)$ is just a self-similar solution of the problem (1.1).

This completes the proof of Theorem 2.6.

\section{Acknowledgment}

This work is supported by Natural Science Foundation of Henan Province Education Commission (no. 2007110013) and the Program for Outstanding Young Teacher in Henan Province (2004-2006). 


\section{References}

[1] T. Cazenave and F. B. Weissler, "The Cauchy problem for the critical nonlinear Schrödinger equation in $H^{s}, "$ Nonlinear Analysis. Theory, Methods \& Applications, vol. 14, no. 10, pp. 807-836, 1990.

[2] J. Ginibre and G. Velo, "On a class of nonlinear Schrödinger equations," Journal of Functional Analysis, vol. 32, pp. 1-71, 1979.

[3] J. Ginibre and G. Velo, "Scattering theory in the energy space for a class of nonlinear Schrödinger equations," Journal de Mathématiques Pures et Appliquées, vol. 64, no. 4, pp. 363-401, 1985.

[4] J. Ginibre and G. Velo, "The global Cauchy problem for the nonlinear Schrödinger equation revisited," Annales de l'Institut Henri Poincaré. Analyse Non Linéaire, vol. 2, no. 4, pp. 309-327, 1985.

[5] M. Nakamura and T. Ozawa, "Low energy scattering for nonlinear Schrödinger equations in fractional order Sobolev spaces," Reviews in Mathematical Physics, vol. 9, no. 3, pp. 397-410, 1997.

[6] T. Cazenave and F. B. Weissler, "Asymptotically self-similar global solutions of the nonlinear Schrödinger and heat equations," Mathematische Zeitschrift, vol. 228, no. 1, pp. 83-120, 1998.

[7] F. Ribaud and A. Youssfi, "Regular and self-similar solutions of nonlinear Schrödinger equations," Journal de Mathématiques Pures et Appliquées, vol. 77, no. 10, pp. 1065-1079, 1998.

[8] H. Pecher and W. von Wahl, "Time dependent nonlinear Schrödinger equations," Manuscripta Mathematica, vol. 27, no. 2, pp. 125-157, 1979.

[9] P. Sjögren and P. Sjölin, “Local regularity of solutions to time-dependent Schrödinger equations with smooth potentials," Annales Academiae Scientiarum Fennicae. Series A I. Mathematica, vol. 16, no. 1, pp. 3-12, 1991.

[10] P. Sjölin, "Regularity of solutions to nonlinear equations of Schrödinger type," Tohoku Mathematical Journal, vol. 45, no. 2, pp. 191-203, 1993.

[11] Y. J. Ye, "The global small solutions for a class of nonlinear Schrödinger equations," Acta Mathematicae Applicatae Sinica, vol. 29, no. 1, pp. 91-96, 2006.

[12] B. Guo and B. Wang, "The global Cauchy problem and scattering of solutions for nonlinear Schrödinger equations in $H^{s}$," Differential and Integral Equations, vol. 15, no. 9, pp. 1073-1083, 2002.

[13] C. X. Miao, "The global strong solution for Schrödinger equation of higher order," Acta Mathematicae Applicatae Sinica, vol. 19, no. 2, pp. 213-221, 1996, Chinese.

[14] C. X. Miao, Harmonic Analysis and Applications to Partial Differential Equations, Science Press, Beijing, China, 1999.

[15] W. Littman, "Fourier transforms of surface-carried measures and differentiability of surface averages," Bulletin of the American Mathematical Society, vol. 69, pp. 766-770, 1963.

[16] F. Ribaud and A. Youssfi, "Self-similar solutions of the nonlinear wave equation," preprint, 2007. 


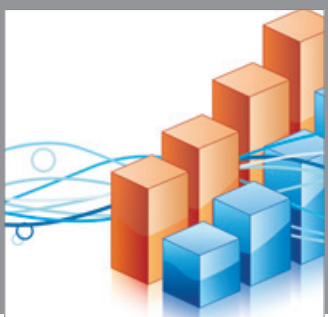

Advances in

Operations Research

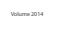

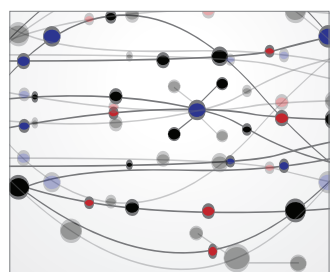

\section{The Scientific} World Journal
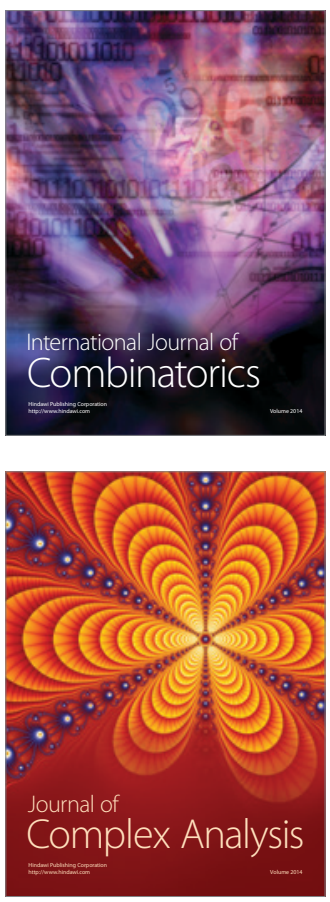

International Journal of

Mathematics and

Mathematical

Sciences
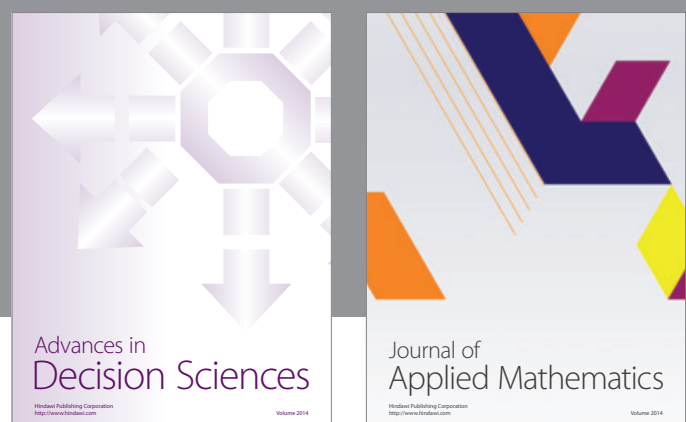

Journal of

Applied Mathematics
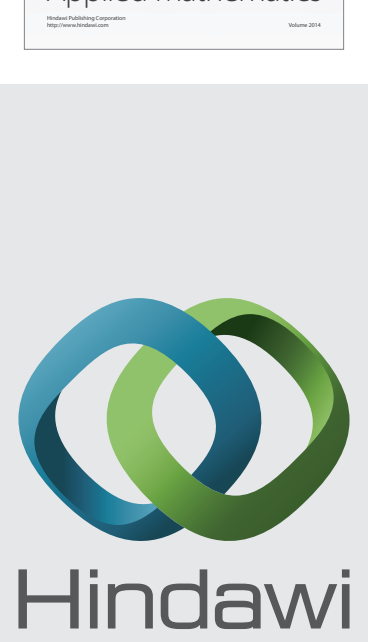

Submit your manuscripts at http://www.hindawi.com
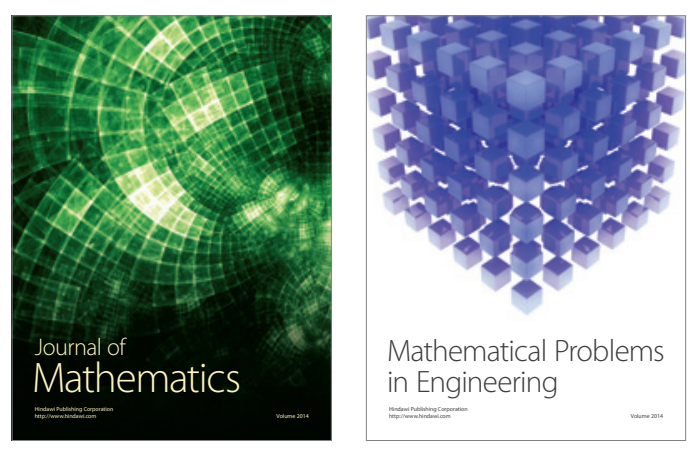

Mathematical Problems in Engineering
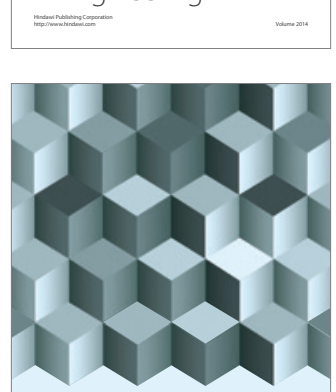

Journal of

Function Spaces
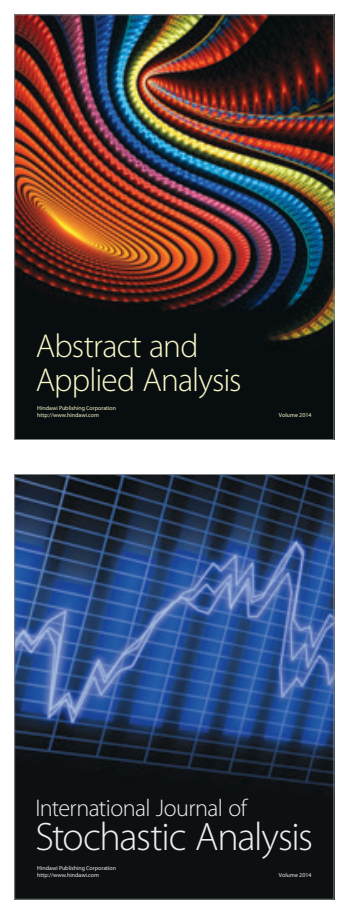

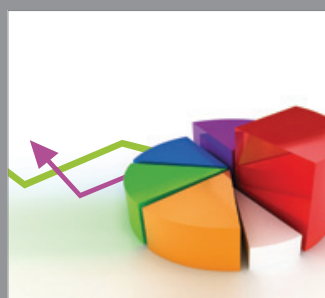

ournal of

Probability and Statistics

Promensencen
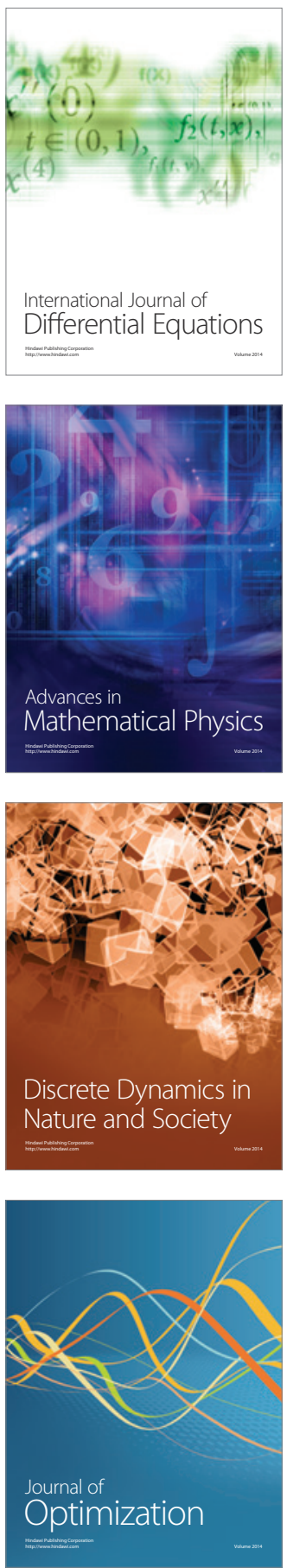